\title{
A Modeling Framework for Decision Support in Periprosthetic Joint Infection Treatment
}

\author{
Vasiliy N. LEONENKO ${ }^{\mathrm{a} 1}$, Yulia E. KALIBERDA ${ }^{\mathrm{a}}$ and Vasiliy A. ARTYUK ${ }^{\mathrm{b}}$ \\ a ITMO University, St. Petersburg, Russia \\ ${ }^{\mathrm{b}}$ Russian Scientific Research Institute of Traumatology and Orthopedics "R.R. Vreden", \\ St. Petersburg, Russia
}

\begin{abstract}
In this paper, we present a framework, which aims at facilitating the choice of the best strategy related to the treatment of periprosthetic joint infection (PJI). The framework includes two models: a detailed non-Markovian model based on the decision tree approach, and a general Markov model, which captures the most essential states of a patient under treatment. The application of the framework is demonstrated on the dataset provided by Russian Scientific Research Institute of Traumatology and Orthopedics "R.R. Vreden", which contains records of patients with PJI occurred after total hip arthroplasty. The methods of cost-effectiveness analysis of treatment strategies and forecasting of individual treatment outcomes depending on the selected strategy are discussed.
\end{abstract}

Keywords. Modeling framework, periprosthetic joint infection, decision support, Markov model, decision tree

\section{Introduction}

In today's aging society with high demands on mobility, arthroplasty is increasingly performed for both elderly and younger patients. The widespread use of replacing the affected joints with artificial ones increases the need for revision arthroplasty (re-THR). Today, periprosthetic joint infection (PJI) is one of the leading indications for revision surgery and by far the most ominous complication in artificial joint patients [1]. Periprosthetic infection is associated with high morbidity and requires complex treatment strategies including multiple surgical revisions and long-term antimicrobial treatment, because the implant as a foreign body increases the pathogenicity of bacteria and the presence of biofilm makes the diagnosis and treatment problematic [2].

A scientific approach to medical practice requires a search for evidence of the efficacy and safety of existing and promising surgical methods for treating PJI. The optimal tool in solving this problem is evidence-based medicine, allowing comparison, generalization and wide practical application of the data obtained [3]. Thus, the need for a clinical and economic analysis of PJI treatment from the standpoint of evidencebased medicine seems to be very relevant. The investigations related to the creation of cost-

${ }^{1}$ Corresponding Author: V.N. Leonenko, ITMO University, Kronverksky Pr. 49A, St. Petersburg, 197101,Russia; E-mail: vnleonenko@itmo.ru 
effective approaches to PJI treatment $[4,5]$ mention the issues connected with the corresponding analysis due to lack of quality studies.

In this paper, we present a framework, which aims at facilitating the choice of the best strategy related to the treatment of PJI. The application of the framework is demonstrated on the dataset provided by Russian Scientific Research Institute of Traumatologyand Orthopedics "R.R. Vreden", which contains records of the patients with PJI occurred after total hip arthroplasty. The methods of cost-effectiveness analysis of treatment strategies and forecasting of individual treatment outcomes depending on the selected strategy are discussed.

\section{Data}

The analyzed data set contains the records of 571 patient who were subjected to a revision total hip replacement (re-THR) in the period of 2000-2020. The patient records were collected in two different ways. A large part of the disease histories (a so-called retrospective group of 405 patients) was taken from the archives, whereas the remaining part (prospective group, 166 patients) was filled in real time by adding data of the patients undergoingtreatment starting from 2014. All the patients were being observed for the possible PJI relapse till the end of 2020. In the retrospective group, the following treatment methods have been applied: resection arthroplasty (RA), revision operation with the preservation of endoprosthesis (re-THR-PE) and two-stage revision total hip replacement with the two consecutive interventions separated by more than 2 months. In the prospective group, new treatment methods were presented, namely, one-stage reTHR and partial re-THR. Also, in the prospective group, the waiting time between surgeries for two-stage revisions did not exceed 2 months. The patients in the prospective group who underwent two- stage re-THR were divided into two subgroups based on their waiting time: 2-3 weeks and 6-8 weeks correspondingly. In many cases, in addition to PJI-related surgeries, additional operations were required due to the relapse of PJI or other issues (postoperativewound hematomas, spacer dislocations, etc.). The recorded data we worked with contains 15 different types of operations, which were divided into three groups: operations, which have no connection with PJI (e.g., endoprosthesis (EP) installation + spacer removal, EPinstallation (no spacer), etc.); first case of PJI or PJI relapse (e.g., debridement, debridement + spacer installation); PJI relapse (e.g., debridement + spacer reinstallation, spacer removal + support osteotomy).

\section{Framework Description}

\subsection{Decision Tree}

As a continuation of our previous research efforts [6], we developed an algorithm for creating and verifying detailed decision trees, which in their turn serve as a base of an imitational discrete-event model. The decision tree is comprised of the transitions between the states, which are attributed to different medical interventions. Each transitionsignifies the change in patient functional capacity and is associated with the treatment costs. The time passed between the transitions is not explicitly taken into account. 
The framework builds trees for a given treatment method based on the subsamples of the corresponding patient records. The calibration procedure algorithm uses a recursive approach and generates tree branches with the transition probabilities as an output. To assess how the results of the sample analysis might be generalized to an independent dataset of patients, we calculate mean values of the transition probabilities along with their interval assessments with the help of bootstrap aggregating technique. A fragment of a decision tree for partial re-THR is shown in Figure 1.

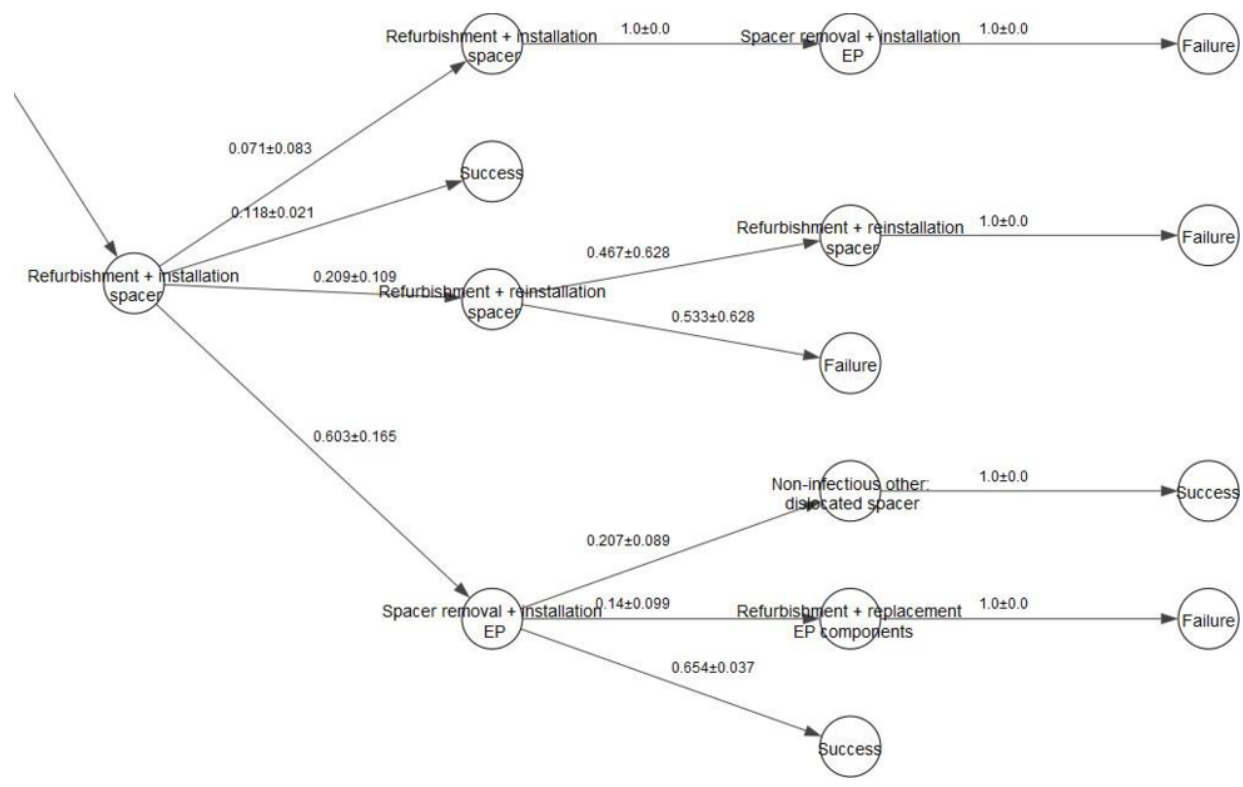

Figure 1. A fragment of the decision tree with confidence intervals for transition probabilities, partial re-THR

The decision trees based on the mean probabilities constitute a base for the discrete event imitational model. Each simulation run consists of generating an individual patient trajectory via Monte-Carlo methods according to the probabilities calculated during the cross-validation procedure. The output of the model includes generated individual trajectories and probability distributions for the treatment states calculated via repetitive simulation runs.

The decision tree has the advantage of detailed description of trajectories, which makes it possible to calculate the statistics of changes in expected expenses and the overall QALY (quality-adjusted life-years, a generic measure of disease burden), related to particular interventions. Our framework supports the assignments of two associated parameters, related to the impact of the intervention, to each branch of the tree. The first parameter is the intervention cost in rubles taken from the list of costs of intervention types. The second parameter is the gained utility of the patient during a fixed timeframe calculated in QALY units. The quantitative outcomes of the treatment in terms of healthcare costs and QALY units gained by the patient might be derived from the decision tree using the following formula: $C=\sum_{i} p_{i} c_{i}$, where $p_{i}$ is the probability of selecting the branch, obtained by cross-validation, $c_{i}$ is the impact measured in either of the two units. 
The interval assessment of $C$ can be calculated using the same formula with left and right boundaries for $p_{i}$ used instead of their mean assessments.

The resulting values of $C_{r u b l}$ and $C_{u}$ might be used to calculate the costs of one QALY unit and compare them between the treatment strategies. QALY units and costs for particular tree branches might be derived from the external sources, for instance, the data of the Center for the Evaluation of Value and Risk in Health which was used in thesimilar calculation from [7]. In the current study, however, we relied on the data provided by Russian Scientific Research Institute of Traumatology and Orthopedics "R.R. Vreden". The operation costs were taken from the disease histories, and the QALY units were assessed based on the EQ-5D indices for each particular patient measured between the subsequent operations [8]. The results of QALY assessment are given in Tables 1, 2.

Table 1. Average QALY for treatment methods in the retrospective group according to the decision trees

\begin{tabular}{|c|c|c|c|}
\hline Treatment method & Re-THR-PE & Two-stage $>2$ months & RA \\
\hline QALY & 1.537 & 2.363 & 0.675 \\
\hline
\end{tabular}

Table 2. Average QALY for treatment methods in the prospective group according to the decision trees

\begin{tabular}{|c|c|c|c|c|}
\hline Treatment method & Two-stage 2-3 weeks & Two-stage 6-8 weeks & One stage & Partial \\
\hline QALY & 1.124 & 0.634 & 1.035 & 1.053 \\
\hline
\end{tabular}

\subsection{Markov Model}

The disadvantage of a decision tree approach is that the tree might become extensively big and intractable in case of big samples of patients, long observation time or big variety of possible interventions. Also, it does not account for the time passed between the model states, which sometimes can be crucial for the accurate cost-effectiveness analysis. As an alternative approach, we developed a Markov model with generalized states. The generalized intervention types assumed in the model are created according to the intervention classification described in Sec. 2. We distinguish PJI-related interventions (PJI or PJI relapse), interventions not related to PJI, and, as a separate intervention type, a second stage intervention for two-stage treatment methods ('Endoprosthesis installation + spacer removal'). The states of the simulated patient are the following: (a) PJI (waiting for the treatment), (b) second stage (no PJI, waiting for the spacer removal in two-stage treatment methods), (c) additional surgeries (waiting for the treatment of a non-PJI issue), (d) observation (no PJI), (e) death. The last state is the absorbing state. The simulation starts from the state 'PJI'. The situations of a first PJI case and a recurrent PJI are not distinguished due to the lack of corresponding data in the records. The time in the model is discrete, with the time step equal to one month.

The transitional probabilities are calculated based on the available patient records using the same bootstrapping procedure as for the decision tree case. An example of the calibrated model is shown in Figure 2. The model makes it possible to generate individual patient trajectories with the consideration of time, which is useful for the calculation of the expected amount of observation time and the hospitalization time - the latter greatly influences the overall treatment costs. 


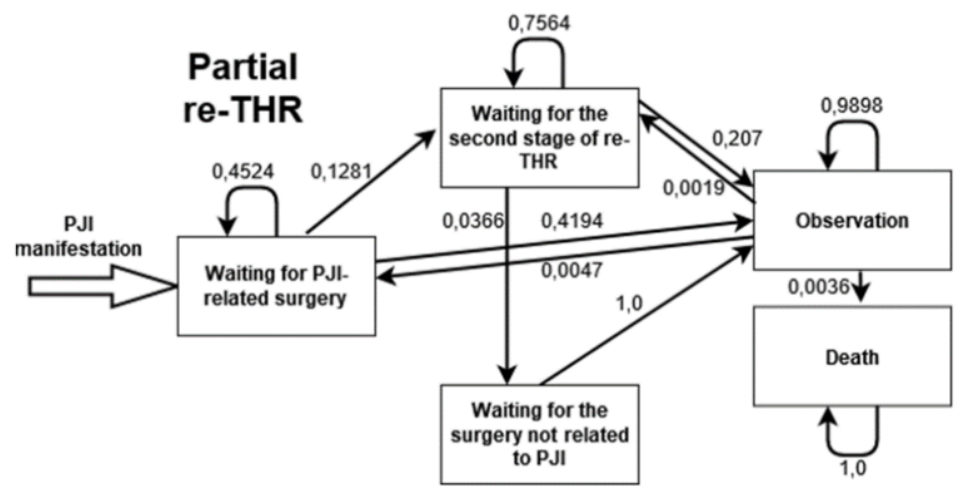

Figure 2. Markov model calibration results for partial re-THR (confidence intervals for the probabilities are omitted)

The treatment impact for a fixed individual patient trajectory is calculated according to the formula $C(t)=\sum_{i} t_{i} c_{i}$, where $c_{i}$ are the monthly costs or QALY units associated with the patient state $i$ in the model, and $t_{i}$ is the patient's time of staying in the state $i$ (the number of months). Unlike in case of the decision tree, accurate numbers of $c_{i}$ (in both rubles and QALY units) are not available due to generalized states used in the model. They could be taken by averaging the costs for the patient state based on the set of particular interventions related to that state and on the experts' opinion. For example, it might be assumed that the ideal state of the patient in terms of QALY units relates to 'Observation' status. The lowest QALY values correspond to 'PJI'. The quality of life of a patient waiting for the second stage or additional surgeries is higher than in case of PJI, but lower than in the 'Observation' state due to corresponding health issues (particularly, the patients waiting for the second stage of the treatment have limited mobility due to spacer installation which badly affects their QALY count). Average QALY values calculated for different methods are shown in Tables 3 and 4. Following expert assumptions, we set the QALY for 'PJI' state equal to 0.35 , for 'Second stage' equal to 0.7 , for'Non-PJI operation' equal to 0.5 , for 'Observation' equal to 0.85 , and for 'Death' equal to 0 .

Table 3. Average QALY for treatment methods in the retrospective group according to the Markov simulations

\begin{tabular}{|c|c|c|c|}
\hline Treatment method & Re-THR-PE & Two-stage $>2$ months & RA \\
\hline QALY & 1.88 & 1.92 & 1.79 \\
\hline
\end{tabular}

Table 4. Average QALY for treatment methods in the prospective group according to the Markov simulations

\begin{tabular}{|c|c|c|c|c|}
\hline Treatment method & Two-stage 2-3 weeks & Two-stage 6-8 weeks & One stage & Partial \\
\hline QALY & 1.925 & 2.055 & 1.99 & 1.952 \\
\hline
\end{tabular}

\section{Discussion}

In this paper, a modeling framework is presented, which aims at facilitating the decision making for healthcare professionals in the area of periprosthetic joint infection treatment. By using two different approaches within one framework, which is the decision tree approach and the Markov modeling approach, we can obtain a detailed static analysis of 
the prospected patient treatment trajectories, depending on the selected strategy, or alternatively perform a dynamic simulation of a patient trajectory of transitions between the generalized states in an imitational model. The former helps to calculate detailed total operational costs and obtained quality of life, their average values and their distributions, whereas the latter gives an opportunity to monitor and forecast the dynamics of costs and QALY units. After an additional verification of the models, specialists of Russian Scientific Research Institute of Traumatology and Orthopedics "R.R. Vreden" will test the proposed framework in clinical practice. Based on the results obtained, we plan to develop this tool further and to apply it for other problems related to PJI treatment. One of the possible framework applications consists in forecasting disease trajectories for patients using their individual characteristics (age, gender, body mass index) as parameters affecting the transition probabilities. Another one is to consider the prediction of PJI cases at the city level, using synthesized populationsas a model input $[9,10]$. The framework coupled with the statistical model of PJI occurrence probability and the synthetic demographic data will make it possible to assess the middle- and long-term expenses of PJI treatment, the hospital occupancy and the prospected potential years of life lost in the urban population depending on the prevalent treatment methods.

\section{Acknowledgement}

This research is financially supported by The Russian Science Foundation, Agreement \#19-11-00326.

\section{References}

[1] Shubnyakov II, Tikhilov RM, DenisovAO, Akhmedilov MA, Cherny AZ, et al. What Has Changed in the Structure of Revision Hip Arthroplasty?. Traumatologyand Orthopedics of Russia, 2019; 25(4):9-27.

[2] Masters EA, Trombetta RP, de Mesy Bentley KL, Boyce BF, Gill AL, et al. Evolving concepts in bone infection: redefining "bio-film", "acute vs. chronic osteomyelitis", "the immune proteome" and "local antibiotic therapy". Bone research, 2019; 7(1):1-18.

[3] Guyatt G, Cairns J, Churchill D, Cook D, Haynes B, et al. Evidence-based medicine: a new approach to teaching the practice of medicine. JAMA,(1992; 268(17):2420-2425.

[4] Li C, Renz N, Trampuz A. Management of periprosthetic joint infection. Hip \& pelvis, 2018; 30(3):138146.

[5] Hernández-Vaquero D, Fernández-Fairen M, Torres A, Menzie AM, Fernández-Carreira JM, et al. Treatment of periprosthetic infections: an economic analysis. The Scientific World Journal, 2013; 2013:821650. doi:10.1155/2013/821650.

[6] Kaliberda YE, Leonenko VN, Artyukh VA. Towards Cost-Effective Treatment of Periprosthetic Joint Infection: From Statistical Analysis to Markov Models. In International Conference on Computational Science (pp. 494-505). Springer, Cham, 2021, June.

[7] Srivastava K., Bozic KJ, Silverton C, Nelson AJ, Makhni EC, Davis JJ. Reconsidering strategies for managing chronic periprosthetic joint infection in total knee arthroplasty: using decision analytics to find the optimal strategy between one-stage and two-stage total knee revision. JBJS, 2019;101(1):14-24.

[8] Dolan P. Modeling valuations for EuroQol health states. Medical care, 1997; 35(11): 1095-1108.

[9] Bates S, Leonenko V, Rineer J, Bobashev G. Using synthetic populations to understand geospatial patterns in opioid related overdose and predicted opioid misuse. Computational and Mathematical Organization Theory, 2019; 25(1):36-47.

[10] Leonenko VN. Analyzing the spatial distribution of acute coronary syndrome cases using synthesizeddata on arterial hypertension prevalence. In International Conference on Computational Science (pp. 483-494). Springer, Cham, 2020, June. 